\title{
Diagnosis and management of lumbar Aspergillus spp. discospondylitis using intraoperative cytology and external stabilization in a dog with disseminated infection
}

\author{
Josep Brocal $^{1 *}$, Fernando Ribas del Río ${ }^{2}$ and Alejandro Luján Feliu-Pascual ${ }^{3}$ \\ ${ }^{1}$ WEAR REFERRALS Veterinary Hospital, Stockton-on-Tees TS21 2ES, UK \\ ${ }^{2}$ CV San Jorge, Luisa Oliver, Sant Jordi, Eivissa 07817, Spain \\ ${ }^{3}$ Aúna Especialidades Veterinarias, Valencia 46980, Spain
}

\begin{abstract}
Background: Discospondylitis is an infection of the intervertebral disc and adjacent vertebral endplates. The infectious agent is most commonly a bacterial organism and fungal causes are uncommon.

Case Description: A 1.5-year-old female entire pug was referred with a 6-week history of right head tilt and progressive non-ambulatory paraparesis. On neurological examination, right facial paralysis and mid and caudal lumbar pain were also detected. Magnetic resonance imaging and computed tomography of the head and spine were performed 3 weeks apart. Findings were consistent with osteolysis of the petrous temporal bone and L2-L3 and L5-L6 vertebral subluxation caused by discospondylitis and osteomyelitis. Disseminated aspergillosis was diagnosed following biopsy and stabilization using an external skeletal spinal fixator. Voriconazol was administered as a medical treatment after surgery. The dog died 3 months later without failure of the construct.

Conclusion: To the authors' knowledge, this is the first report using an external fixator for fungal lumbar discospondylitis. The use of an external skeletal spinal fixator should be considered when choosing the surgical technique.
\end{abstract}

Keywords: Aspergillus, Cytology, Discospondylitis, Dog, External fixation.

\section{Introduction}

Discospondylitis is an infection of the intervertebral disc and adjacent vertebral endplates, with the hematogenous spread being the most common route of infection (Tipold and Stein, 2010). Fungal causes of discospondylitis are uncommon (Hurov et al., 1978). Both medical and surgical treatments for dogs with discospondylitis have been described (Hurov et al., 1978; McKee et al., 1990; Auger et al., 2000; Cabassu and Moissonnier, 2007; Renwick et al., 2010). Surgical treatment mainly involves decompression of the spinal cord and curettage, allowing sample collection for culture. Stabilization of the spine for discospondylitis has been rarely reported in veterinary literature.

To the author's knowledge, no previous report in veterinary literature has described the use of an external skeletal spinal fixator (ESSF) for the treatment of fungal discospondylitis. The use of an ESSF for the treatment of discospondyltis has only been previously reported in the lumbosacral junction (Auger et al., 2000).

\section{Case details}

A 1.5-year old, $7.5 \mathrm{~kg}$, black female pug was evaluated with a 3-week history of lumbar pain, difficulty jumping, and right head tilt. A magnetic resonance imaging study of the head and the lumbar spine was initially performed with a $0.2 \mathrm{~T}$ magnet (Vet-MR; Esaote, Genova, Italy) at the local veterinary surgeon. A focal poorly demarcated area of contrast enhancement (gadoterate meglumine, Dotarem; Guerbet Roissy CdGCedex, France) in the right temporal bone and adjacent temporal muscle was found. In the lumbar spine, cortical bone destruction and heterogeneous contrast uptake affecting both L2-L3 and L5-L6 intervertebral spaces and surrounding paraspinal musculature, consistent with discospondylitis, osteomyelitis, and myositis were detected. Following progressive deterioration and lack of response to broad-spectrum antibiotics and nonsteroidal anti-inflammatory drugs, the dog was referred. At the time of referral, bilateral exophthalmus, right non-responsive miosis, right facial paralysis causing a large corneal ulcer, right head tilt, left horizontal nystagmus, non-ambulatory paraparesis, and mid and caudal lumbar spinal pain suggested a multi-focal localization affecting the right temporal area and lumbar spine.

Hematology and comprehensive biochemistry and radiographs of the thorax and abdomen were performed. Abnormalities on hematology and serum biochemistry included a slight neutrophilia and monocytosis, and a mild elevation in urea and total protein. The patient was sedated with methadone $(0.3$ 
$\mathrm{mg} / \mathrm{kg} \mathrm{IV})$ and acepromazine $(0.02 \mathrm{mg} / \mathrm{kg} \mathrm{IV})$, induced with propofol $(1 \mathrm{mg} / \mathrm{kg} \mathrm{IV})$ and general anesthesia maintained with isofluorane. Plain radiographs of the entire vertebral column and thorax, and a computed tomography (CT) scan (Toshiba Aquilion; Zoetermeer, The Netherlands) of the lumbar spine following intra-thecal injection of iomeprol $0.3 \mathrm{ml} /$ $\mathrm{kg}$ (Iomeron $250 \mathrm{mg} / \mathrm{ml}$; Bracco.p.a, Milan, Italy) at L5-L6 subarachnoid space were performed. Findings included lysis of the caudal endplate of L2 and cranial endplate of L3, and a pathologic fracture of the caudal metaphysis of L2 causing subluxation (Fig. 1). These bony changes were associated with isoattenuating material causing ventral extradural spinal cord compression at that level. The above description was consistent with discospondylitis, osteomyelitis and a pathological fracture with subsequent vertebral subluxation, and epidural empyema. Similar but less severe lesions were found in L5-L6 with minimal spinal cord compression.

The CT images of the head revealed an extensive but circumscribed lytic lesion in the pars petrosa of the right temporal bone, leading to a pathologic fracture of the right temporomandibular joint condyle. The right masseter muscle enhanced focally (iomeprol at a dose of $400 \mathrm{mg} / \mathrm{kg} \mathrm{IV}$ ), and the right tympanic bullae had a ventral isoattenuating meniscus (Fig. 2). No abnormalities were detected in thoracic radiographs or abdominal CT.

The L2-L3 vertebral lesion was decompressed and curettaged preserving the dorsal lamina, articular facets, and pedicles via a bilateral dorsal approach. Intraoperative samples were taken for cytology, histopathology, and bacterial and fungal culture. Gentamicin-impregnated collagen sponges were placed in the space created.

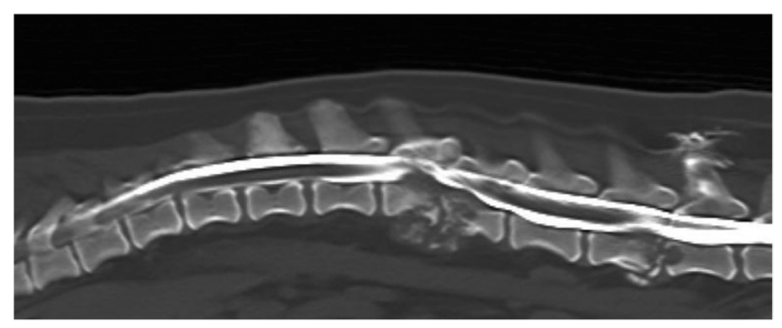

Fig. 1. Computed tomography reconstructed sagittal image of the thoracic and lumbar spine illustrating lysis on the caudal endplate of L2 and cranial endplate of L3, with irregular and poorly defined margins; an irregular periosteal reaction of the lateral aspects of the vertebral bodies and vertebral arch, and a pathologic fracture of the caudal metaphysis of L2 causing subluxation. These bony changes are associated with isoattenuating material along the ventral and ventrolateral aspect of the vertebral canal over the vertebral bodies of L2 and L3, causing ventral extradural spinal cord compression. Similar but less severe lesions were found affecting L5-L6 with minimal ventral compression of the spinal cord.
The subluxacion was reduced manually and stabilized using a bilateral and biplanar ESSF. A total of six $2.5 \mathrm{~mm}$ positive-partially-threaded pins, eight Meynard clamps and three connecting rods were implanted. The positivepartially-threaded pins were inserted in the vertebral bodies of L1 and L4, avoiding contact with the affected vertebrae. Immediate post-operative radiographs were obtained confirming reduced subluxation and realigned vertebrae (Fig. 3).

In-house cytology of impression smears made of the material collected showed structures compatible with fungal hyphae amongst neutrophils and fibroblasts (Fig. 4).

Postoperatively, clindamicin (15 mg/kg q12h IV), enrofloxacin (5 mg/kg q12h IV), ampicilin (22 mg/ $\mathrm{kg}$ q12h IV), meloxicam (0.1 mg/kg q24h SC), and fluconazol ( $5 \mathrm{mg} / \mathrm{kg}$ q12h PO) were administered while culture results were pending. The right corneal ulcer was treated with topical antibiotics and artificial tears. Histopathology results showed multiple areas of granulomatous-necrotizing swelling and the presence of fungal structures with hyphae characteristics after Grocott's stain.

Five days after the surgery the dog was discharged with slightly decreased muscle tone of the pelvic limbs, but intact nociception and tail movements.

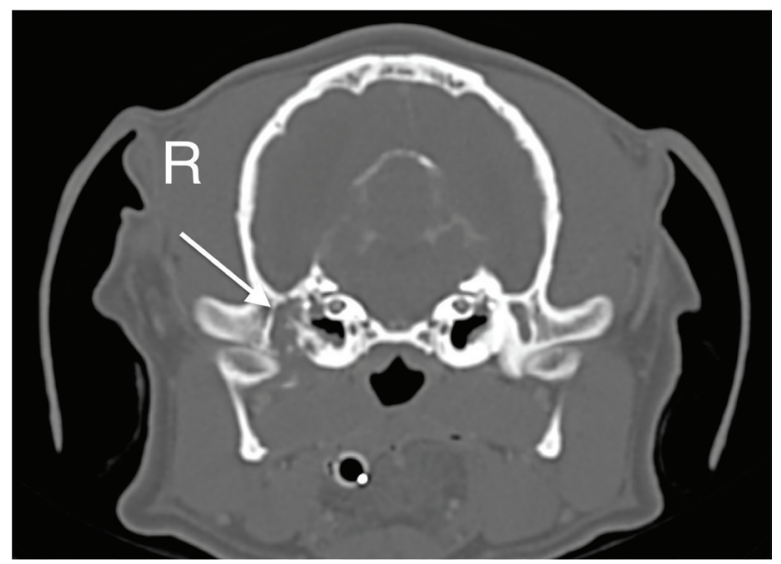

Fig. 2. Computer tomography transverse image of the head showing an extensive but circumscribed lytic lesion in the pars petrosa of the right temporal bone and a pathologic fracture of the right temporomandibular joint condyle with caudodorsal displacement of the distal fragment.

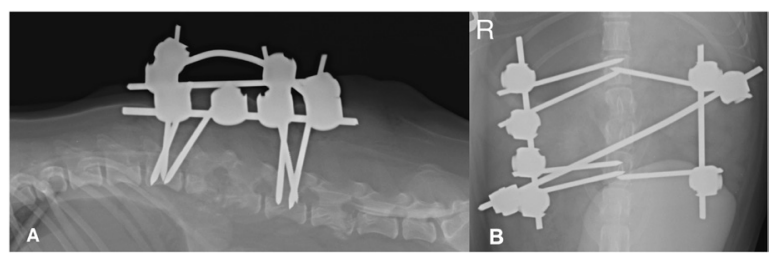

Fig. 3. Lateral (A) and dorso-ventral (B) postoperative radiograph of the lumbar spine showing the bilateral and biplanar external spinal skeletal fixator after curettage and manual reduction of the L2-L3 subluxation. 
Bacterial cultures were negative and the fungal organism was classified as Aspergillus terreus. No susceptibility test was performed. Antibiotic therapy was withdrawn, fluconazole was discontinued and voriconazol (Vfend; PfizerLtd, Kent, United Kingdom) was started at an extrapolated dose from human medicine of $3 \mathrm{mg} / \mathrm{kg}$ q12h PO.

Radiographic follow-up by the referring veterinary surgeon two months later revealed lack of vertebral fusion and progressive osteolysis with new lesions at L1-L2, L3-L4, and L4-L5 intervertebral disc spaces (Fig. 5). Regardless, the vertebral column remained realigned without failure of the construct.

The dog died approximately 3 months after the surgery following multi-organ failure. No loosening of the pins was noticed and post-mortem examination was not granted.

\section{Discussion}

Fungal discospondylitis is usually characterized by multiple distant lesions, indicating a systemic infection and reinforcing a hematogenous spread. Aspergillus terreus and Aspergillus fumigatus are commonly involved (Schultz et al., 2008).

Surgical treatment of discospondylitis is reserved for medical failure, moderate and severe neurological deficits, considerable vertebral bony destruction, and vertebral instability (Gage, 1975; Gilmore, 1987; Ozuna and Delamarter, 1996). The aims are curettage, obtain biological samples, decompress the spinal cord, and stabilize to achieve bony fusion.

Internal and external fixation devices have been used for the treatment of vertebral osteomyelitis in human literature (Jeanneret and Magerl, 1994; Dai et al.,

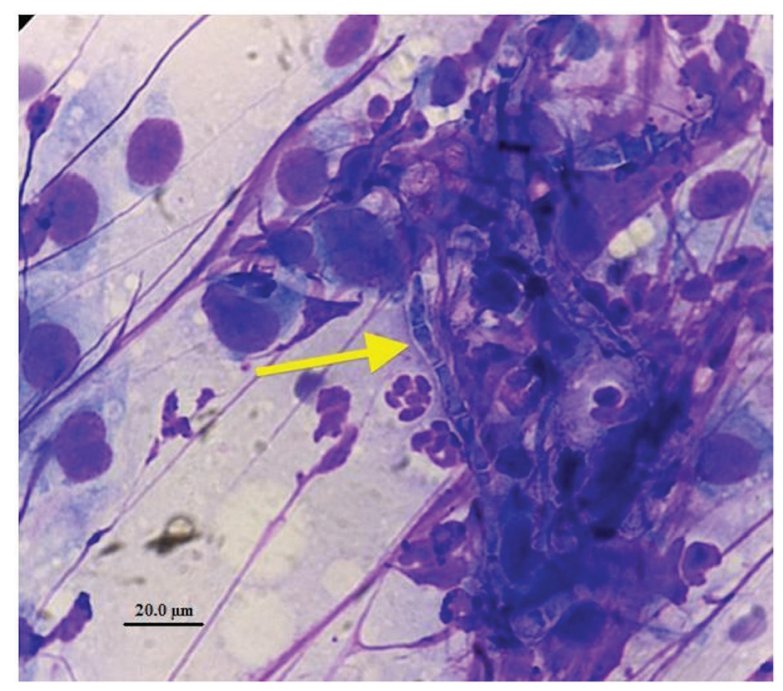

Fig. 4. Intraoperative cytology with Diff-Quick staining made of material collected during curettage at L2-L3 and showing fungal hyphae (arrow), non-degenerated neutrophils, and cells with a fusiform aspect and a prominent nucleus consistent with fibroblasts.

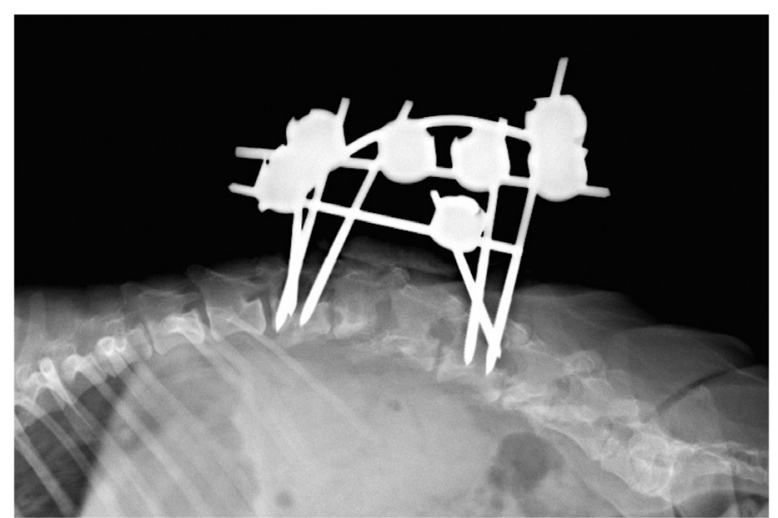

Fig. 5. Lateral radiograph of the thoracic and lumbar spine illustrating lack of vertebral fusion and increased osteolytic lesions at L2-L3 two months after surgery. New osteolytic lesions were observed at the endplates of the adjacent vertebra to L1-L2, L3-L4, and L4-L5 intervertebral disc spaces.

2008). In dogs, polymethylmethacrylate and screws have been used in the treatment of thoracic (Cabassu and Moissonnier, 2007) and lumbosacral (Renwick et al., 2010; Font et al., 2013) bacterial discospondylitis, but not in the lumbar spine. Applying cement and/ or screws to an infected area may pose a risk, but antibiotic-loaded cement can be successfully used (Cabassu and Moissonnier, 2007; Renwick et al., 2010; Font et al., 2013). A distraction-fusion technique using transarticular threaded pins across L7-S1, and bone graft, also reported satisfactory results in four canine cases (McKee et al., 1990; Font et al., 2013).

The use of an ESSF has been previously used for the treatment of vertebral fractures (Lanz et al., 2000) and lumbosacral instability caused by bacterial discospondylitis (Auger et al., 2000). Its use was assessed in a canine unstable spinal model with spinal disarticulation between L3-L4. Mechanical properties comparable to more commonly used polymethylmethacrylate/ pin internal fixations techniques were identified. The study supports the biomechanical stability ESSF of the canine lumbar spine (Walker et al., 2002).

The authors opted for ESSF due to the presence of extensive osteomyelitis, spinal cord compression, and subluxation. To prevent infection, the positivepartially-threaded pins were inserted in the vertebral bodies of L1 and L4, avoiding the infected sites. Had fusion been achieved after infection resolution, implant removal would have been easier compared to internal fixation.

Intraoperative diagnosis using the smear technique cytology has been previously reported (Vernau et al., 2001; Font et al., 2014). A prompt presumptive diagnosis allowed the initiation of antifungal treatment while waiting for biopsy and culture results. The technique may provide information during the 
procedure allowing the neurosurgeon to take informed decisions.

A variety of antifungal agents have been used for the treatment of disseminated aspergillosis in dogs (Schultz et al., 2008). Fluconazol was chosen initially because of its availability and broad spectrum. Because it appears less effective against filamentous fungi such as Aspergillus spp., it was changed for voriconazole following culture results (Greene, 2012), as it combines satisfactory systemic antifungal effects, high oral bioavailability and good bone penetration in humans (Koehler et al., 2014).

Fungal osteomyelitis in dogs has been reported in axial and appendicular skeleton (Smith et al., 2000), but there is no previous report affecting the petrous bone and causing facial paralysis and head tilt. Other cranial nerves abnormalities have been previously described in dogs with systemic mycosis (Watt et al., 1995; Schultz et al., 2008). No sample was taken from the petrous bone, but the multiple distant osteomyelitic lesions suggested a disseminated fungal infection.

Despite voriconazole treatment, our dog deteriorated progressively and never regained ambulation. The temporomandibular joint fracture caused pain; the vertebral osteolytic lesions increased, and episodes of diarrhea and hyporexia became frequent. The dog died 3 months after diagnosis. The 2 months' followup imaging findings suggested the vertebral column remained aligned without failure of the construct, as so was at the time of death. The stability of ESSF in the lumbar region has been proven elsewhere (Walker et al., 2002).

The use of an ESSF might provide stabilization of the vertebral column in cases of fungal or bacterial discospondylitis and should be considered an option when choosing the surgical technique.

\section{Conflict of interest}

The authors declare that there is no conflict of interest. Author contributions

J.B.: concept/design, acquisition of data, drafting of the manuscript, assembled figures, critical revision of the manuscript, and approval of the article.

F.R.: acquisition of data, critical revision of the manuscript, and approval of the article.

A.L: concept/design, acquisition of data, drafting of the manuscript, assembled figures, critical revision of the manuscript, and approval of the article.

\section{References}

Auger, J., Dupuis, J., Quesnel, A. and Beauregard, G. 2007. Surgical treatment of lumbosacral instability caused by discospondylitis in four dogs. Vet. Surg. 29(1), 70-80.

Cabassu, J. and Moissonnier, P. 2007. Surgical treatment of a vertebral fracture associated with a haematogenous osteomyelitis in a dog. Vet. Comp. Orthop. Traumatol. 20(3), 227-230.
Dai, L.Y., Chen, W.H. and Jiang, L.S. 2008. Anterior instrumentation for the treatment of pyogenic vertebral osteomyelitis of thoracic and lumbar spine. Eur. Spine J. 17, 1027-1034.

Font, C., Mascort, J. and Lujan, A. 2013. Surgical internal stabilization in three canine cases with incomplete response to conservative management. In Proceedings of the Southern European Veterinary Conference, October, Barcelona, Spain.

Font, C., Mascort, J., Márquez, M., Esteban, C., Sánchez, D., Durall, N., Pumarola, M. and Luján, A. 2014. Paraparesis as initial manifestation of a Prototheca zopfii infection in a dog. J. Small Anim. Pract. 55(5), 283-286.

Gage, E.D. 1975. Treatment of discospondylitis in the dog. J. Am. Vet. Med. Assoc. 166, 1164-1169.

Gilmore, D.R. 1987. Lumbosacral diskospondylitis in 21 dogs. J. Am. Anim. Hosp. Assoc. 23(1), 57-61.

Greene, G.E. 2012. Antifungal chemotherapy. In Infectious diseases of the dog and the cat, 4th ed. Greene, G.E., St. Louis: Elsevier Saunders, pp: 579-588.

Hurov, L., Troy, G. and Turnwald, G. 1978. Diskospondylitis in the dog: 27 cases. J. Am. Vet. Med. Assoc. 173(1), 275-281.

Jeanneret, B. and Magerl, F. 1994. Treatment of osteomyelitis of the spine using percutaneous suction/irrigation and percutaneous external spinal fixation. J. Spinal Disord. 7(3), 185-205.

Koehler, P., Tacke, D. and Cornely, O.A. 2014. Aspergillosis of bones and joints - a review from 2002 until today. Mycoses 57(6), 323-335.

Lanz, O.I., Jones, J.C. and Bergman, R. 2000. Use of an external fixator to correct spinal fracture/ luxation and instability in three dogs. Vet. Neurol. Neurosurg. 2(1).

McKee, W.M., Mitten, R.W. and Labuc, R.H. 1990. Surgical treatment of lumbosacral discospondylitis by a distraction-fusion technique. J. Small Anim. Pract. 31(1), 15-20.

Ozuna, R.M. and Delamarter, R.B. 1996. Pyogenic vertebral osteomyelitis and postsurgical disc space infections. Orthop. Clin. North Am. 27, 87-94.

Renwick, A.I.C., Dennis, R. and Gemmill, T.J. 2010. Treatment of lumbosacral discospondylitis by surgical stabilisation and application of a gentamicin-impregnated collagen sponge. Vet. Comp. Orthop. Traumatol. 23(4), 266-272.

Schultz, R.M., Johnson, E.G., Wisner, E.R., Brown, N.A., Byrne, B.A. and Sykes, J.E. 2008. Clinicopathologic and diagnostic imaging characteristics of systemic Aspergillosis in 30 dogs. J. Vet. Intern. Med. 22, 851-859.

Smith, A.N., Spencer, J.A., Stringfellow, J.S., Vygantas, K.R. and Welch, J.A. 2000. Disseminated infection with phialemonium obovatum in a German 
Shepherd Dog. J. Am. Vet. Med. Assoc. 216(5), 708-712.

Tipold, A. and Stein, V.M. 2010. Inflammatory diseases of the spine in small animals. Vet. Clin. North Am. Small Anim. Pract. 40, 871-879.

Vernau, K.M., Higgins, R.J., Bollen, A.W., Jimenez, D.F., Anderson, J.V., Koblik, P.D. and LeCouteur, R.A. 2001. Primary canine and feline nervous system tumors: intraoperative diagnosis using the smear technique. Vet. Pathol. 38, 47-57.

Walker, T.M., Pierce, W.A. and Welsh, R.D. 2002. External Fixation of the Lumbar Spine in a Canine Model. Vet. Surg. 31, 181-188.

Watt, P.R., Robins, G.M., Galloway, A.M. and O'Boyle, D.A. 1995. Disseminated opportunistic fungal disease in dogs: 10 cases (1982-1990). J. Am. Vet. Med. Assoc. 207, 67-70. 